\title{
Anomalous Dimension in the Solution of the Barenblatt's Equation
}

\author{
Cheng Chen \\ Institute of Plasma Physics, Academia Sinica, \\ Hefei, Anhui, 230031, P.R. China \\ and \\ G. Cheng \\ CCAST (World Laboratory), P.O. Box 8730, Beijing 100080, P.R. China \\ Fundamental Physical Center, USTC., Hefei, Anhui, 230026, P.R. China*
}

\begin{abstract}
A new method is presented to obtain the anomalous dimension in the solution of the Barenblatt's equation. The result is the same as that in the renormalization group (RG) approach. It gives us insight on the perturbative solution of the Barenblatt's equation in the RG approach. Based on this discussion, an improvement is made to take into account, in more complete way, the nonlinear effect, which is included in the Heaviside function in higher orders. This improved result is better than that in RG approach.
\end{abstract}

\section{Introduction}

*Mailing address 
Renormalization and the renormalization group (RG) method are powerful mathematical physics tool in quantum field theory and condensed matter physics. ${ }^{1,2}$ Recently it has been found that there are also important applications in dynamical process, asymptotic analysis and perturbation theory. ${ }^{3-5}$ The fractal and chaos behaviors have been studied with the RG approach ${ }^{3}$. The introduction of the intermediate asymptotic idea is very useful. It can help us to search for the connection between the similarity, fractal and anomalous dimension of the RG theory ${ }^{3}$. But there are still ambiguities in some aspects. For example, in quantum field theory and in dynamical theory, it is well known that the $\mathrm{RG}$ approach can improve the results of the perturbation calculation. ${ }^{1-5}$ It can remove the divergence and lead to higher leading order behavior from a lower order perturbation calculation. It is also clear that the divergence and the renormalization are physical requirements for some theories. But some of the divergences occur without apparent physical reasons. One question involves the improvement that arise from the RG perturbative calculations. In this paper we study this problem by analyzing a typical example.

Barenblatt's equation has many good characteristics. It has strong physical background and appears in many different disciplines. ${ }^{6}$ It has been studied by applying the method of similarity analysis. It can be solved in terms of the parabolic cylinder functions in the intermediate asymptotic region. Its perturbation solution is divergent and has anomalous dimension in its asymptotic behavior. It is renormalizable and can be treated with the renormalization and RG approach ${ }^{3,4}$. But there are still some ambiguities as mentioned above.

In this paper we study improvements on the perturbation solution of the Barenblatt's equation by renormalization and RG treatment and, attempt 
to expose some general principles through the discussion of this example. Based on the above analysis, an improved result is obtained by considering the nonlinear effect, which is included in the Heaviside function in higher orders, in more complete way.

\section{Anomalous dimension calculation by the RG method}

In this section, we repeat some results that are well known, for consistency in notation and discussion style.

We consider the Barenblatt's equation in one space dimension

$$
\partial_{t} u(x, t)-\frac{1}{2} \partial_{x}^{2} u(x, t)=\frac{\varepsilon}{2} \theta\left(-\partial_{t} u(x, t)\right) \partial_{x}^{2} u(x, t),
$$

with symmetrical initial condition. It describes a nonlinear diffusive process with non-conservative 'mass'.

Introduce a change of variables

$$
\xi=\xi(x, t)=\frac{x}{\sqrt{t+l^{2}}} \quad \text { and } \quad T=T(x, t)=t+l^{2} .
$$

Then eq.(1) reads

$\partial_{T} u(\xi, T)-\frac{\xi}{2 T} \partial_{\xi} u(\xi, T)-\frac{1}{2 T} \partial_{\xi}^{2} u(\xi, T)=\frac{\varepsilon}{2 T} \theta\left(\left(-\partial_{T}+\frac{\xi}{2 T} \partial_{\xi}\right) u(\xi, T)\right) \partial_{\xi}^{2} u(\xi, T)$.

We use an initial condition of $u(\xi, T)$ as

$$
u\left(\xi, l^{2}\right)=\frac{m_{0}}{\sqrt{2 \pi l^{2}}} \exp \left(-\frac{\xi^{2}}{2}\right) .
$$

It corresponds to the initial condition for $u(x, t)$ in the variables $x$ and $t$ as follows:

$$
u(x, 0)=\frac{m_{0}}{\sqrt{2 \pi l^{2}}} \exp \left(-\frac{x^{2}}{2 l^{2}}\right) .
$$


For a general diffusion equation, there is a finite solution under this initial condition. In this case, the initial condition is singular when $l^{2}$ approaches zero as a $\delta$-function. $m_{0}$ is a finite constant. But it is not so in the case of Barenblatt's equation ${ }^{3,4,6}$. It must be renormalized to get a finite solution. The $m_{0}$ is no longer a constant when the $l^{2}$ changes. It becomes infinite when the support of the initial state approaches zero. We will discuss that in the end of this section.

Introducing the expansion

$$
u(\xi, T)=u_{0}(\xi, T)+\varepsilon u_{1}(\xi, T)+\cdots \cdot \cdot
$$

one has a sequence of equations:

$$
\begin{gathered}
2 T \partial_{T} u_{0}(\xi, T)-\xi \partial_{\xi} u_{0}(\xi, T)-\partial_{\xi}^{2} u_{0}(\xi, T)=0 \\
2 T \partial_{T} u_{1}(\xi, T)-\xi \partial_{\xi} u_{1}(\xi, T)-\partial_{\xi}^{2} u_{1}(\xi, T)=\theta\left(\left(-\partial_{T}+\frac{\xi}{2 T} \partial_{\xi}\right) u_{0}(\xi, T)\right) \partial_{\xi}^{2} u_{0}(\xi, T),
\end{gathered}
$$

and so on.

Furtherly, we assume that the initial condition of the $u_{0}(\xi, T)$ is:

$$
u_{0}\left(\xi, l^{2}\right)=\frac{m_{0}}{\sqrt{2 \pi l^{2}}} \exp \left(-\frac{\xi^{2}}{2}\right) .
$$

Then the initial condition of the $u_{n}(\xi, T)$ is:

$$
u_{n}\left(\xi, l^{2}\right)=0 \quad n \geq 1 .
$$

The solution of the zero-order approximation is:

$$
u_{0}(\xi, T)=\frac{m_{0}}{\sqrt{2 \pi T}} \exp \left(-\frac{\xi^{2}}{2}\right) .
$$

Since one has

$$
\left(-\partial_{T}+\frac{\xi}{2 T} \partial_{\xi}\right) u_{0}(\xi, T)=\left(\frac{1}{2 T}-\frac{\xi^{2}}{2 T}\right) u_{0}(\xi, T),
$$


one obtains

$$
\theta\left(\left(-\partial_{T}+\frac{\xi}{2 T} \partial_{\xi}\right) u_{0}(\xi, T)\right)=\theta\left(1-\left|\xi^{2}\right|\right) .
$$

This is the Heaviside function of the Barenblatt's equation in its lowest order. We can write the first-order equation as:

$$
2 T \partial_{T} u_{1}(\xi, T)-\xi \partial_{\xi} u_{1}(\xi, T)-\partial_{\xi}^{2} u_{1}(\xi, T)=\theta\left(1-\left|\xi^{2}\right|\right) \partial_{\xi}^{2} u_{0}(\xi, T) .
$$

From the similarity analysis we know that the solution of the Barenblatt's equation can be written in a form of variable separation:

$$
u(\xi, T)=F(T) G(\xi) .
$$

It is adequate when we are only interested in the behavior of the solution in the intermediate asymptotic region.

Expanding

$$
F(T)=F_{0}(T)+\varepsilon F_{1}(T)+\cdots \cdot
$$

and

$$
G(\xi)=G_{0}(\xi)+\varepsilon G_{1}(\xi)+\cdots \cdot
$$

we have

$$
u_{0}(\xi, T)=F_{0}(T) G_{0}(\xi)
$$

and

$$
u_{1}(\xi, T)=F_{0}(T) G_{1}(\xi)+F_{1}(T) G_{0}(\xi) .
$$

We now take

$$
F_{0}(T)=\frac{m_{0}}{\sqrt{2 \pi T}} \quad \text { and } \quad G_{0}(\xi)=\exp \left(-\frac{\xi^{2}}{2}\right) .
$$

The solution in the first-order approximation is

$$
u^{(1)}(\xi, T)=F_{0}(T) G_{0}(\xi)+\varepsilon\left(F_{0}(T) G_{1}(\xi)+F_{1}(T) G_{0}(\xi)\right) .
$$

It tells us that the approximation solution not can be expressed in the form of variable separation in finite order of the perturbative theory. But we will see that the RG solution is in this form. Thus, we shall pursue along this line and accept a corresponding approximation, so that we can use the variable separation solution. 
Inserting eq.(19) into eq.(14) yields

$2 T\left(F_{1}^{\prime} G_{0}+F_{0}^{\prime} G_{1}\right)-\xi\left(F_{1} G_{0}^{\prime}+F_{0} G_{1}^{\prime}\right)-\left(F_{1} G_{0}^{\prime \prime}+F_{0} G_{1}^{\prime \prime}\right)=\theta(1-|\xi|) F_{0} G_{0}^{\prime \prime}$,

where

$$
F_{i}^{\prime}=\frac{d}{d T} F_{i}(T), \quad G_{i}^{\prime}=\frac{d}{d \xi} G_{i}(\xi), \quad \text { and } \quad G_{i}^{\prime \prime}=\frac{d^{2}}{d \xi^{2}} G_{i}(\xi), \quad i=0,1 .
$$

Now, we search for the solution of the $F_{1}(T)$. Integrate this equation with respect to the $\xi$ from $-\infty$ to $+\infty$ and let

$$
\int_{-\infty}^{\infty} G_{1}(\xi) d \xi=a .
$$

After simple integral calculation and the application of the conditions

$$
\lim _{\xi \rightarrow \pm \infty} \xi G_{1}(\xi)=0 \quad \text { and } \quad \lim _{\xi \rightarrow \pm \infty} G_{1}^{\prime}(\xi)=0
$$

one obtains

$$
2 a F_{1}(T) T F_{0}^{\prime}(T)+2 \sqrt{2 \pi} T F_{1}^{\prime}(T)+a F_{0}(T)+\sqrt{2 \pi} F_{1}(T)=-\frac{2}{\sqrt{e}} F_{0}(T) .
$$

Substituting $F_{0}(T)=m_{0} / \sqrt{2 \pi T}$ and $F_{0}^{\prime}(T)=-F_{0}(T) / 2 T$ into eq.(26), we get

$$
T F_{1}^{\prime}(T)+\frac{1}{2} F_{1}(T)+\frac{1}{\sqrt{2 \pi e}} F_{0}(T)=0 .
$$

The solution of eq. (27) is

$$
F_{1}(T)=-F_{0}(T) \beta \ln \frac{T}{l^{2}}
$$

where

$$
\beta=\frac{1}{\sqrt{2 \pi e}}
$$

For obtaining this solution, we have made use of the initial condition

$$
F_{n}\left(l^{2}\right)=0 \quad n \geq 1 .
$$


The first-order approximation of $F(T)$ is

$$
F^{(1)}(T)=F_{0}(T)\left(1-\alpha \ln \frac{T}{l^{2}}\right)
$$

where

$$
\alpha=\varepsilon \beta=\frac{\varepsilon}{\sqrt{2 \pi e}} .
$$

It is the anomalous dimension and is well known in the solution of the Barenblatt's equation in the intermediate asymptotic region by application of RG $\operatorname{approach}^{3,4}$.

In the following discussion, we keep the $G(\xi)$ in its zero-order approximation without considering the higher-order corrections. That is to put $G_{n}(\xi)=0, n \geq 1$. Then the solution of the first order is

$$
u^{(1)}(\xi, T)=F_{0}(T) G_{0}(\xi)\left(1-\alpha \ln \frac{T}{l^{2}}\right) .
$$

It has been proved that this approximation does not have any effect on the function $F^{(1)}(T)$ when we make the RG treatment. We will discuss the space behavior in a separate paper.

For getting a finite but non-zero asymptotic solution, the 'mass'

$$
m_{0}=\int_{-\infty}^{\infty} d x u(x, t)
$$

not can remain a constant. It is necessary to approach infinity when the support of the initial function approaches zero. To obtain a finite solution of Barenblatt's equation, the $u(x, 0)$ must be more singular than the $\delta$-function. It is different from the diffusive equation in regular cases. Moreover, the values of the non-zero order terms are not small, but instead, very large in the intermediate asymptotic region. The renormalization is required. It is well known that the renormalization and RG approach not only remove the divergence but also improve the perturbation expansion solution to higher leading order. ${ }^{1-5}$

Let

$$
m_{0}=m_{R}(\mu) Z\left(\mu, l^{2}\right)
$$

and

$$
Z\left(\mu, l^{2}\right)=1+\alpha \ln \frac{\mu}{l^{2}} .
$$


From the work of Goldenfeld et al, ${ }^{3,4}$ we know that the result of the renormalization and RG treatment is:

$$
u_{R}^{(1)}(\xi, T)=\frac{m_{R}\left(l^{2}\right)}{\sqrt{2 \pi T}}\left(\frac{l^{2}}{T}\right)^{\alpha} \exp \left(-\frac{\xi^{2}}{2}\right) .
$$

It is easy to check that the initial condition, the boundary conditions and the connection condition are all satisfied when the $l^{2}$ is taken in non-zero value. But we must remember that the renormalization mass will approach the infinite when $l^{2} \rightarrow 0$, in order to keep the solution finite. Usually we require

$$
m_{R}\left(l^{2}\right) \times l^{2 \alpha}=\text { finite. }
$$

There is an explanation for the improvement of the $\mathrm{RG}$ approach. ${ }^{7}$ But the whole case is not very clear yet. We will discuss some aspects of it in following sections.

\section{Anomalous dimension calculation by summing $F(T)$ to the infinite order}

Now we discuss the improvement to the perturbative solution of the Barenblatt's equation in the RG approach. We do this by using the approximation suggested in the previous section. We are especially interested in the behavior of the intermediate asymptotic region, in which $\xi$ and $t$ are far from the zero point but not in infinite. We shall prove that the result of the renormalization and $\mathrm{RG}$ approach is equivalent to the infinite sum of the series of $F(T)$.

We assume the solution of eq.(3) in a form of variable separation in any order. We have known that it is not a suitable form when the approximation order $n$ is finite. But, the higher the approximation order is taken, the better the proposed form is. As the first step, we keep the dependence on space in its lowest-order. Thus we set

$$
u_{n}(\xi, T)=f_{n}(T) u_{0}(\xi, T) .
$$

At the same time, we also neglect a part of the nonlinear effects. We keep the Heaviside function in its lowest order as $\theta\left(1-\left|\xi^{2}\right|\right)$. Therefore we have the form of perturbation expansion of the Barenblatt's equation.

$$
2 T \partial_{T} u_{n}(\xi, T)-\xi \partial_{\xi} u_{n}(\xi, T)-\partial_{\xi}^{2} u_{n}(\xi, T)=
$$




$$
=\theta\left(\left(-\partial_{T}+\frac{\xi}{2 T} \partial_{\xi}\right) u_{0}(\xi, T)\right) \partial_{\xi}^{2} u_{n-1}(\xi, T) \quad n \geq 1 .
$$

We will prove that the RG anomalous dimension is just the result of summing up the $F(T)$ series to infinite order in the above approximation.

Inserting eqs.(13) and (39) into eq.(40) yields

$$
2 T u_{0}(\xi, T) \frac{d}{d T} f_{n}(T)=\theta\left(1-\left|\xi^{2}\right|\right) f_{n-1}(T) \partial_{\xi}^{2} u_{0}(\xi, T) \quad n \geq 1 .
$$

The initial condition

$$
u_{n}\left(\xi, l^{2}\right)=0 \quad n \geq 1
$$

becomes

$$
f_{n}\left(l^{2}\right)=0 \quad n \geq 1
$$

When $n=1$, one has

$$
2 T u_{0}(\xi, T) \frac{d}{d T} f_{1}(T)=\theta\left(1-\left|\xi^{2}\right|\right) \partial_{\xi}^{2} u_{0}(\xi, T) .
$$

Integrating it with respect to $\xi$ from $-\infty$ to $+\infty$ gives

$$
T \frac{d}{d T} f_{1}(T)=-\frac{1}{\sqrt{2 \pi e}}=-\beta
$$

Using $f_{n}\left(l^{2}\right)=0, n \geq 1$, the solution of eq.(45) is

$$
f_{1}(T)=-\frac{1}{\sqrt{2 \pi e}} \ln \frac{T}{l^{2}}=\ln \left(\frac{T}{l^{2}}\right)^{-\beta} .
$$

When $n=2$, one has

$$
2 T u_{0}(\xi, T) \frac{d}{d T} f_{2}(T)=\theta\left(1-\left|\xi^{2}\right|\right) f_{1}(T) \partial_{\xi}^{2} u_{0}(\xi, T)
$$

Doing the same integration as we have done to the eq.(44), we obtain

$$
2 T \frac{m_{0}}{\sqrt{T}} \frac{d}{d T} f_{2}(T)=f_{1}(T) \int_{-1}^{1} d \xi \partial_{\xi}^{2} u_{0}(\xi, T)
$$


Due to the identity

$$
2 T \frac{m_{0}}{\sqrt{T}} \frac{d}{d T} f_{1}(T)=\int_{-1}^{1} d \xi \partial_{\xi}^{2} u_{0}(\xi, T),
$$

we have

$$
\frac{d}{d T} f_{2}(T)=f_{1}(T) \frac{d}{d T} f_{1}(T) .
$$

Making use of $f_{n}\left(l^{2}\right)=0, n \geq 1$, we obtain

$$
f_{2}(T)=\frac{1}{2}\left[f_{1}(T)\right]^{2} .
$$

Using the principle of mathematical induction, we now prove that

$$
f_{m}(T)=\frac{1}{m !}\left[f_{1}(T)\right]^{m} .
$$

Suppose that eq.(52) is true for $m=n-1$, that is to say,

$$
f_{n-1}(T)=\frac{1}{(n-1) !}\left[f_{1}(T)\right]^{n-1}
$$

We are going to prove that it is true for $m=n$. Integrating eq.(41) with respect to $\xi$ from $-\infty$ to $+\infty$ gives

$$
2 T \frac{m_{0}}{\sqrt{T}} \frac{d}{d T} f_{n}(T)=f_{n-1}(T) \int_{-1}^{1} d \xi \partial_{\xi}^{2} u_{0}(\xi, T) .
$$

Due to eq.(49), one has

$$
\frac{d}{d T} f_{n}(T)=f_{n-1}(T) \frac{d}{d T} f_{1}(T) .
$$

Substituting eq.(53) into it gives

$$
\frac{d}{d T} f_{n}(T)=\frac{1}{(n-1) !}\left[f_{1}(T)\right]^{n-1} \frac{d}{d T} f_{1}(T) .
$$

Because $f_{n}\left(l^{2}\right)=0$, the solution of eq. (56) is

$$
f_{n}(T)=\frac{1}{n !}\left[f_{1}(T)\right]^{n} .
$$


It is true for $m=1$ and $m=2$. Thus we have proved the proposition for any $m$.

In the infinite limit, we have

$$
u(\xi, T)=\left[1+\sum_{n=1}^{\infty} \frac{\varepsilon^{n}}{n !}\left[f_{1}(T)\right]^{n}\right] u_{0}(\xi, T) .
$$

Inserting eq.(46) into it yields

$$
u(\xi, T)=\left[1+\sum_{n=1}^{\infty} \frac{1}{n !}\left[-\varepsilon \beta \ln \frac{T}{l^{2}}\right]^{n}\right] u_{0}(\xi, T),
$$

that is

$$
u(\xi, T)=\exp \left[-\varepsilon \beta \ln \frac{T}{l^{2}}\right] u_{0}(\xi, T)=\left[\frac{T}{l^{2}}\right]^{-\alpha} u_{0}(\xi, T)
$$

where

$$
\alpha=\varepsilon \beta=\frac{\varepsilon}{\sqrt{2 \pi e}},
$$

which is just the anomalous dimension given by the renormalization and RG approach.

\section{An improvement on the calculation of the anoma- lous dimension}

In the previous section, we have kept the Heaviside function and the function $G(\xi)$ in their lowest order in our calculation of the $F(T)$ to higher orders. It has led a partial loss of the nonlinear effect which is included in the Heaviside function. Now we show that the calculation of the anomalous dimension can be improved by the infinite summing of the function $F(T)$ without the approximation of keeping the Heaviside function in its lowest order to take account of a part of the lost nonlinear effect. Now we still keep the solution in the form of variable separation as eq.(15), but only let the function $G(\xi)$ in it lowest order. We shall use a trick that makes an analytic treatment possible.

Take the approximation solution obtained in the previous section

$$
u(\xi, T)=\left(\frac{T}{l^{2}}\right)^{-\alpha} u_{0}(\xi, T) .
$$


as an initial solution. After inserting it into the Heaviside function, we solve the $u_{n}(\xi, T)$ and search for its infinite sum. Thus we obtain the first iterative result.

We put the first iterative solution into the Heaviside function to solve the equation secondly.

In this way, we search for the limit of the iterative solution to infinite. If the limit exists, we can obtain the solution.

An important observation is that the form of the solution remains unchanged in the iterative process.

$$
u^{(k)}(\xi, T)=\left(\frac{T}{l^{2}}\right)^{-\alpha^{(k)}} u_{0}(\xi, T) .
$$

The only change is the value of the anomalous dimension $\alpha^{(k)}$. It simplifies the analysis considerably.

We rewrite $\alpha$ as $\alpha^{(0)}$

$$
\alpha=\varepsilon \beta=\frac{\varepsilon}{\sqrt{2 \pi e}} \rightarrow \alpha^{(0)}=\varepsilon \beta^{(0)} .
$$

The $\alpha^{(k)}=\varepsilon \beta^{(k)}$ represents the results after the $k$ th iteration.

We still need to solve the problem by summing up the infinite expansion series, in each step of the iterative process.

Putting eq.(63) into the Heaviside function of the $n$ th-order equation, we obtain the $n$th order and $(k+1)$ th iteration equation

$$
\begin{gathered}
2 T u_{0}(\xi, T) \frac{d}{d T} f_{n}^{(k+1)}(T)= \\
\theta\left(\left(-\partial_{T}+\frac{\xi}{2 T} \partial_{\xi}\right)\left(\left(\frac{T}{l^{2}}\right)^{-\alpha^{(k)}} u_{0}(\xi, T)\right)\right) f_{n-1}^{(k+1)}(T) \partial_{\xi}^{2} u_{0}(\xi, T) .
\end{gathered}
$$

where the super-index $(k)$ represents the result of the $k$ th iteration and the sub-index $n$ represents the $n$th order in the perturbation expansion. The initial condition remains in the form of eq.(43).

When $k=1$ and $n=1$, one has

$2 T u_{0}(\xi, T) \frac{d}{d T} f_{1}^{(1)}(T)=\theta\left(\left(-\partial_{T}+\frac{\xi}{2 T} \partial_{\xi}\right)\left(\left(\frac{T}{l^{2}}\right)^{-\alpha^{(0)}} u_{0}(\xi, T)\right)\right) \partial_{\xi}^{2} u_{0}(\xi, T)$, 
where $f_{0}^{(k)}(T)=1$ has been used. Because of the identity

$$
\left(-\partial_{T}+\frac{\xi}{2 T} \partial_{\xi}\right)\left(\left(\frac{T}{l^{2}}\right)^{-\alpha^{(0)}} u_{0}(\xi, T)\right)=\left[2 \alpha^{(0)}-\left(\xi^{2}-1\right)\right] \frac{1}{2 T}\left(\frac{T}{l^{2}}\right)^{-\alpha^{(0)}} u_{0}(\xi, T),
$$

one has

$$
\theta\left(\left(-\partial_{T}+\frac{\xi}{2 T} \partial_{\xi}\right)\left(\left(\frac{T}{l^{2}}\right)^{-\alpha^{(0)}} u_{0}(\xi, T)\right)\right)=\theta\left(2 \alpha^{(0)}+1-\xi^{2}\right) .
$$

Substituting it into eq.(66) and integrating it with respect to $\xi$ from $-\infty$ to $+\infty$ yields

$$
\begin{gathered}
2 T \frac{m_{0}}{\sqrt{T}} \frac{d}{d T} f_{1}^{(1)}(T)=\int_{-\infty}^{\infty} \theta\left(2 \alpha^{(0)}+1-\xi^{2}\right) \partial_{\xi}^{2} u_{0}(\xi, T) \\
=\frac{m_{0}}{\sqrt{2 \pi T}}\left[-2 \sqrt{\left(1+2 \alpha^{(0)}\right)} \exp \left(-\frac{1+2 \alpha^{(0)}}{2}\right)\right],
\end{gathered}
$$

that is,

$$
\frac{d}{d \ln T} f_{1}^{(1)}(T)=\frac{1}{\sqrt{2 \pi}}\left[-\sqrt{\left(1+2 \alpha^{(0)}\right)} \exp \left(-\frac{1+2 \alpha^{(0)}}{2}\right)\right] .
$$

Using $f_{1}^{(1)}\left(l^{2}\right)=0$, we can obtain the solution of eq.(70)

$$
f_{1}^{(1)}(T)=\frac{-\sqrt{\left(1+2 \alpha^{(0)}\right)}}{\sqrt{2 \pi e^{\left(1+2 \alpha^{(0)}\right)}}} \ln \frac{T}{l^{2}}=-\beta^{(1)} \ln \frac{T}{l^{2}},
$$

where

$$
\beta^{(1)}=\frac{\sqrt{\left(1+2 \alpha^{(0)}\right)}}{\sqrt{2 \pi e^{\left(1+2 \alpha^{(0)}\right)}}} .
$$

When $k=1$ and $n=2$, one has

$$
2 T u_{0}(\xi, T) \frac{d}{d T} f_{2}^{(1)}(T)=\theta\left(2 \alpha^{(0)}+1-\xi^{2}\right) f_{1}^{(1)}(T) \partial_{\xi}^{2} u_{0}(\xi, T)
$$


Integrating it with respect to $\xi$ from $-\infty$ to $+\infty$ yields

$$
\frac{d}{d T} f_{2}^{(1)}(T)=f_{1}^{(1)}(T) \frac{d}{d T} f_{1}^{(1)}(T) .
$$

Making use of $f_{n}^{(1)}\left(l^{2}\right)=0, n \geq 2$, the solution of eq.(74) is

$$
f_{2}^{(1)}(T)=\frac{1}{2}\left[f_{1}^{(1)}(T)\right]^{2} .
$$

Using the principle of mathematical induction, it is easy to prove that

$$
f_{m}^{(1)}(T)=\frac{1}{m !}\left[f_{1}^{(1)}(T)\right]^{m}
$$

Suppose it is true for $m=n-1$, that is to say

$$
f_{n-1}^{(1)}(T)=\frac{1}{(n-1) !}\left[f_{1}^{(1)}(T)\right]^{n-1} .
$$

We are going to prove that it is true for $m=n$. We know that

$$
2 T u_{0}(\xi, T) \frac{d}{d T} f_{n}^{(1)}(T)=\theta\left(2 \alpha^{(0)}+1-\xi^{2}\right) f_{n-1}^{(1)}(T) \partial_{\xi}^{2} u_{0}(\xi, T) .
$$

Integrating it with respect to $\xi$ from $-\infty$ to $+\infty$ gives

$$
\frac{d}{d T} f_{n}^{(1)}(T)=f_{n-1}^{(1)}(T) \frac{d}{d T} f_{1}^{(1)}(T) .
$$

Substituting eq.(77) into it yields

$$
\frac{d}{d T} f_{n}^{(1)}(T)=\frac{1}{(n-1) !}\left[f_{1}^{(1)}(T)\right]^{n-1} \frac{d}{d T} f_{1}^{(1)}(T) .
$$

On account of $f_{n}^{(1)}\left(l^{2}\right)=0$, one can solve eq.(80) and obtain

$$
f_{n}^{(1)}(T)=\frac{1}{n !}\left[f_{1}^{(1)}(T)\right]^{n} .
$$

We have proved that it is true for $m=1$ and $m=2$. Thus the proposition is true for any $m$. 
Now we sum the infinite series

$$
u^{(1)}(\xi, T)=\left[1+\sum_{n=1}^{\infty} \frac{\varepsilon^{n}}{n !}\left[f_{1}^{(1)}(T)\right]^{n}\right] u_{0}(\xi, T) .
$$

Inserting the expression of $f_{1}^{(1)}(T)$ into it, we obtain

$$
u^{(1)}(\xi, T)=\left[1+\sum_{n=1}^{\infty} \frac{1}{n !}\left[-\varepsilon \beta^{(1)} \ln \frac{T}{l^{2}}\right]^{n}\right] u_{0}(\xi, T) .
$$

That is

$$
u^{(1)}(\xi, T)=\left[\frac{T}{l^{2}}\right]^{-\alpha^{(1)}} u_{0}(\xi, T)
$$

where

$$
\alpha^{(1)}=\varepsilon \beta^{(1)} \quad \text { and } \quad \beta^{(1)}=\frac{\sqrt{\left(1+2 \alpha^{(0)}\right)}}{\sqrt{2 \pi e^{\left(1+2 \alpha^{(0)}\right)}}} .
$$

The $\alpha^{(1)}$ is the anomalous dimension corrected to the first-order of the $F(T)$. Now, we have finished the first iterative calculation.

When we make the $k$ th iteration, the process is the same as the $(k-1)$ th one. The only difference is that we will use $\beta^{(k)}$ in place of the $\beta^{(k-1)}$. Then it is easy to prove that

$$
u^{(k)}(\xi, T)=\left[\frac{T}{l^{2}}\right]^{-\alpha^{(k)}} u_{0}(\xi, T),
$$

where

$$
\alpha^{(k)}=\varepsilon \beta^{(k)} \quad \text { and } \quad \beta^{(k)}=\frac{\sqrt{\left(1+2 \alpha^{(k-1)}\right)}}{\sqrt{2 \pi e^{\left(1+2 \alpha^{(k-1)}\right)}}} .
$$

The $\alpha^{(k)}$ is the anomalous dimension corrected to the $k$ th order of the $F(T)$.

If the iterative process is convergent, the limit exists. Let $\lim _{k \rightarrow \infty} \beta^{(k)}=$ $\beta^{(\infty)}$, which leads to

$$
\beta^{(\infty)}=\frac{\sqrt{\left(1+2 \varepsilon \beta^{(\infty)}\right)}}{\sqrt{2 \pi e^{\left(1+2 \varepsilon \beta^{(\infty)}\right)}}}
$$

Then one has

$$
u^{(\infty)}(\xi, T)=\left[\frac{T}{l^{2}}\right]^{-\alpha^{(\infty)}} u_{0}(\xi, T)
$$


where

$$
\alpha^{(\infty)}=\varepsilon \beta^{(\infty)}
$$

and

$$
\alpha^{(\infty)}=\frac{\varepsilon \sqrt{\left(1+2 \alpha^{(\infty)}\right)}}{\sqrt{2 \pi e^{\left(1+2 \alpha^{(\infty)}\right)}}}
$$

It is obvious that eq.(91) has finite solution, which is the anomalous dimension corrected by considering the nonlinear effect of $F(T)$ to infinite order.

The justification of the iterative method and its initial value is that the solution is convergent and satisfies the Barenblatt's equation in our approximation.

We can compare the improved result with the numerical one given by Barenblatt and the result obtained by RG approach as shown in Fig.1. The improved result $\alpha^{(\infty)}$ is better than $\alpha^{(0)}$.

\section{Conclusion}

We have calculated the anomalous dimension by summing the $F(T)$ to infinite order. When we keep the Heaviside function and the space dependent function $G(\xi)$ in their lowest order, the anomalous dimension is the same as the $\mathrm{RG}$ result. When the approximation of the Heaviside function in its lowest order is removed, the value of the anomalous dimension can be improved. It is expressed in eq.(91) and Fig.1.

\section{Acknowledgments}

G. Cheng is supported in part by the National Science Foundation in China and by the NDSTPR. Foundation in China. The authors would like to thank Miss J.L. Zhou, Dr. Zhang Yang and Dr. Han Lang for their help in this paper. 


\section{References}

[1] N.N. Bogoliubov and D.V. Shirkov, Introduction to the Theory of Quantized Fields, third edition, John Wiley \& Sons, Inc., New York, (1980); J. Zinn-Justin, Quantum Field Theory and Critical Phenomena, Clarendon Press., Oxford, (1989).

[2] M. Gell-Mann and F.T. Low, Phys. Rev. 95, 1300 (1964); K. Symanzik, Comm. Math. Phys., 18, p. 227 (1970); C.G. Callan, Phys. Rev., D2, p.1541 (1970); K.G. Wilson, Phys. Rev. B4, p.3174 and p.3184 (1971); K.G. Wilson, and J. Kogut, Phys. Rep. C12, 75 (1974).

[3] N. Goldenfeld, Lectures on Phase Transitions and the Renormalization Group, Addison-Wesley Publishing Company, (1992); R.J. Creswick, H.A. Farach and C.P. Poole, JR., Introduction to Renormalization Group Methods in Physics, Wiley, New York, (1992).

[4] N. Goldenfeld, O. Martin and Y. Oono, J. Sci. Comp. 4, p.4 (1989); N. Goldenfeld, O. Martin, Y. Oono, and F. Liu, Phys. Rev. Lett., 64, p.1361 (1990); Lin-Yuan Chen, N. Goldenfeld, and Y. Oono, Phys. Rev. A44, p.6544 (1991); J. Bricmont and A. Kupiainen, Comm. Math. Phys. 150, p.193 (1992).

[5] Lin-Yuan Chen, N. Goldenfeld, and Y. Oono, Phys. Rev. Lett., 73, p.1311 (1994); Lin-Yuan Chen, N. Goldenfeld, Y. Oono, and G. Paquette, Physica, A204, p.111 (1994); G. Paquette, Lin-Yuan Chen, and N. Goldenfeld, Y. Oono, Phys. Rev. Lett., 72, p.76 (1994). G. Caginalp, Phys.Rev. E53, p.66 (1996).

[6] G.I. Barenblatt, Similarity, Self-Similarity and Intermediate Asymptotics, Consultants Bureeu, New York, (1979).

[7] T. Kunihiro, Prog. Theor. Phys., 97, p.503 (1995). 